\title{
Risk Multidimensional Measure of Complex Accident System
}

\author{
Song Wang \\ Equipment Engineering College \\ Engineering University of Armed Police Force of China \\ Xi'an Shaanxi \\ onesoon@163.com
}

\begin{abstract}
In order to improve complex system safety level, it must be based on an accurate measure of risk, from the perspective of complex accident system, extracted the risk multidimensional status indicators. Then improved the extenics method, the correlation function and affiliation between multidimensional risk indicators to classic matterelement region (risk level region) and festival field matterelement region (risk indicators value region) were considered. The geometric mean and arithmetic mean of single indicator risk degree was integrated to calculate the final comprehensive risk degree. The proposed method can effectively improve the objectivity and accuracy of the risk measure.
\end{abstract}

Keywords-complex system; risk measure; extenics method; risk indicator

\section{INTRODUCTION}

Safety accidents go with the development of human being, along with the enhancing of safety, reliability and automatization of modern complex system, its complexity and nonlinear coupling increasing at the same time, which makes the changing of accident inherence, and even the designer can't understand all the potential interaction within the system. Existing accident theory and models which aim at mechanical systems and simple mechanicalelectronical systems can't effectively explain the dynamics and nonlinear interactions between system components in modern complex systems, so develop and perfect the new systemic accident model is singularly significant.

Highly technological systems such as aviation, maritime, air traffic control, telecommunications, nuclear power plants, defence and aerospace, chemical and petroleum industry, and healthcare and patient safety are exceedingly becoming more complex[1,2]. Accident models provide a conceptualisation of the characteristics of the accident, which typically show the relation between causes and effects [3]. They explain why accidents occur, and are used as techniques for risk assessment during system development, and for post hoc accident analysis to study the causes of the occurrence of an accident [4].

Based on systemic science and complexity science, author synthetically used multi-subjects theory and methods, such as Control Science and Engineering, System Engineering, Entropy, Complex Network, et al., encircled the cognize-restrict model to outspread the study, adopted the research path of constructed accident system modelcognized accident system risk behavior (risk emergence and transfer)-distinguished and restricted key causation nodes, put forward a new systemic accident models which aim at complex system safety accident advance prevention, emergence control and accident analysis whole process. The conclusions have positive sense in perfecting accident causation theory and preventing future safety accident.

Main presupposition lying behind complex system science is that it's not possible to describe, or understand, behavior of a system by only knowing how it's constituent components behave, integrity of the system causes the emergence. Parts of the behavior of a whole is also in connections that are formed between it's components. This is quite opposite from the more traditional reductionist approach in which basic components are analyzed and then the behavior of the whole is induced based on that analysis.

Major accidents keep occurring that seem preventable and that have similar systemic causes. Too often, we fail to learn from the past and make inadequate changes in response to accidents [5]. It is important to understand the causes of accidents in complex systems in order to enhance the safety of such systems, and to develop preventative strategies to mitigate the occurrence of future similar accidents [4]. Risk assessment is a structured sciencebased process to estimate the likelihood and severity of risk with attendant uncertainty [6]. Most traditional risk assessment method are based on one dimensional or two dimensional, and the results are not objectivity, so the risk assessment method should be expanded to multidimensional angle [7], this paper will improve the Matter-clement model to provide a risk multidimensional measure of complex accident system, and offer the measure method for risk entropy.

\section{TRADITIONAL RISK ASSESSMENT METHOD}

A risk measure is a function, say $R($.$) , that transforms$ the risks into a real number, which can correspond to the extra cost necessary for keeping the investment position under acceptable risk (a position with an acceptable future net worth). This acceptable risk is often decided by a supervisor such as [8]:

1. a regulator who takes into account the unfavorable states when allowing a risky position that may draw on the resources of the government-for example as a guarantor of last resort;

2. an exchange's clearing firm, which has to make good on the promises to all parties of transactions being securely completed;

3. an investment manager who knows that his firm has basically given to its traders an exit option in which the 
strike "price" consists in being fired in the event of big trading losses on one's position.

Risk assessment is the determination of quantitative or qualitative value of risk related to a concrete situation and a recognized threat (also called hazard). Quantitative risk assessment requires calculations of two components of risk: the magnitude of the potential loss, and the probability that the loss will occur.

Different areas have different understanding of risk, therefore, formed different risk measurement methods. In financial risk measure field, there are sensitivity method [9], volatility law, coherent risk measure method and so on.

\section{A. One dimensional risk assessment}

In measure of project schedule time, two main variables should be considered, namely the total duration mean $\mathrm{E}(\mathrm{T})$ and variance of the total duration $\mathrm{V}(\mathrm{T})$, and the schedule risk can be expressed as:

$$
R_{T}=\frac{\sqrt{V(T)}}{E(T)}
$$

Where $R_{T}$ is the schedule risk, equation (1) is actually considered only the actual duration deviation from the expected mean, which belonging to one dimensional risk assessment.

\section{B. Two dimensional risk assessment}

Based on risk probability and risk consequences, the two dimensional risk assessment model can be expressed as:

$$
R_{2}=f(P, C)
$$

Where, $R_{2}$ is the two-dimensional risk, $P$ means the probability of risk event, $C$ means the possible consequences of risk event.

\section{Multidimensional risk assessment}

Despite the two-dimensional risk measure is more objective than the one-dimensional model, and Williams T.M. [7] consider that the use of $P$ and $C$ as a risk expectations value conceal some important information, and may produce incorrect results, and he also believes that use only $P$ and $C$ to describe the risk is not enough, other risk aspects should be considered, such as risk predictability, risk randomicity, risk cognitive capability and so on. Thus, the two-dimensional risk measure must be to promote the multi-dimensional [10].

\section{EXTENICS AND ITS IMPROVEMENT}

Extenics is a new discipline which solves contradiction problems. It studies the extensibility of things and the laws and methods of exploitation and innovation with formalized model. This paper briefly introduces its study objects, theoretical frame, methodological system, application, scientific significance, utility value and prospect of development [12].

We consider an object $v$ and the quantity of $N$ about $c$. These $N, c$, and $v$ are called three essential factors, Here $v=c(N)$ reflects the relationship between the quantity and quality of an object. If an object has multiple characters or more than one of them are needed to be listed out, then a multi-dimensional (for example, $n$-dimensions) matter element can be defined as:

$$
R=\left(N, c_{i}, v_{i}\right)=\left[\begin{array}{c}
R_{1} \\
R_{2} \\
\vdots \\
R_{n}
\end{array}\right]=\left[\begin{array}{ccc}
N & c_{1} & v_{1} \\
& c_{2} & v_{2} \\
& \vdots & \vdots \\
& c_{n} & v_{n}
\end{array}\right]
$$

Where $c_{1}, c_{2}, \ldots, c_{n}$ are the $n$th characteristics, and $v_{l}, v_{2}, \ldots, v_{n}$ are the corresponding characteristic value, obviously $v_{i}(i=1,2, \ldots, n)$ can have different representation.

Corresponds to multidimensional risk structure measure, $N$ represents the risk, $c_{l}, c_{2}, \ldots, c_{n}$ are risk multidimensional measure index, $v_{1}, v_{2}, \ldots, v_{n}$ are value of multidimensional measure index, then $R$ in formula (3) is the risk element model. For risk probability, $v_{i}$ is determined value, for risk consequences, $v_{i}$ can be interval value. Combining qualitative and quantitative methods of matter-element theory, which can be used to integrated measure the problem of multidimensional risk index.

\section{A. Distance}

In extenics theory, the relation between a point and a region is considered. The distance between two points has been defined in the classical mathematics, i.e., $\rho(x, y)=$ $|\mathrm{x}-\mathrm{y}|$, is the distance between points $\mathrm{x}$ and $\mathrm{y}$. In order to establish an associative function, the distance between a point and a finite region should be defined. Let $\mathrm{x}$ be any point in the domain of real, $(-\infty,+\infty)$, and $\langle x, y\rangle, X_{0}=<a$, b> are two regions in the domain, then the distance between point $\mathrm{x}$ and the region $\mathrm{X}_{0}$ can be defined as [11]

$$
\rho\left(x, X_{0}\right)=\left|x-\frac{a+b}{2}\right|-\frac{1}{2}(b-a)
$$

And the distance between region $\langle\mathrm{x}, \mathrm{y}\rangle$ and the region $\mathrm{X}_{0}$ can be defined as

$$
\begin{aligned}
\rho\left(<x, y>, X_{0}\right) & =\frac{1}{2}\left(\rho\left(x, X_{0}\right)+\rho\left(y, X_{0}\right)\right) \\
= & \frac{1}{2}\left(\left|x-\frac{a+b}{2}\right|+\left|y-\frac{a+b}{2}\right|-(b-a)\right)
\end{aligned}
$$

\section{B. Value of the position}

Put the multidimensional risk index into $m$ standard mode or risk level, describe them as multidimensional matter-element model as follow, which are also the "classical field" in Extenics, then

$$
\begin{aligned}
& R_{j}=\left[\begin{array}{ccc}
N_{j} & c_{1} & V_{1 j} \\
& c_{2} & V_{2 j} \\
& \vdots & \vdots \\
& c_{n} & V_{n j}
\end{array}\right] \\
& =\left[\begin{array}{ccc}
N_{j} & c_{1} & <a_{1 j}, b_{1 j}> \\
& c_{2} & <a_{2 j}, b_{2 j}> \\
\vdots & \vdots \\
& c_{n} & <a_{n j}, b_{n j}>
\end{array}\right] j=1,2, \cdots, m
\end{aligned}
$$

Where, $R_{j}$ is the $j$ th $(j=1,2, \ldots, m)$ risk level matter-element model, $N_{j}$ is the $j$ th risk level, $c_{i}$ is the $i$ th risk measure index, $V_{i j}=<a_{i j}, b_{i j}>$ is the magnitude range of $c_{i}$ under risk level $N_{j}$, which are also $X_{0}$ in formula (4) and (5). 
Risk multidimensional measure index allowed ranges value matter-element model, constitute "festival field" in extenics, there

$$
R_{p}=\left[\begin{array}{ccc}
N_{p} & c_{1} & V_{1 p} \\
& c_{2} & V_{2 p} \\
\vdots & \vdots \\
& c_{n} & V_{n p}
\end{array}\right]=\left[\begin{array}{ccc}
N_{p} & c_{1} & <c_{1 p}, d_{1 p}> \\
& c_{2} & <c_{2 p}, d_{2 p}> \\
& \vdots & \vdots \\
& c_{n} & <c_{n p}, d_{n p}>
\end{array}\right]
$$

Where, $R_{p}$ means the risk matter-element model "festival field", $V_{i p}=<c_{i p}, d_{i p}>$ is the allowed ranges value of measurement index $c_{i}$, satisfied $V_{i j}$ included in the $V_{i p}$.

In addition to considering the relation between a point and a region, the relation between two regions and the relation between a point and two regions should also be considered. The relation between a point and two regions can be expressed by a value of the position $\mathrm{D}$, defined as follows: Let $X_{0}=\langle a, b\rangle$ as the risk level matterelement model value interval, $X=\langle c, d\rangle$ as festival field matter-element model value interval, then the value of the position $\mathrm{D}\left(x, X_{0}, X\right)$ is the relation between the point $x$ and regions $X_{0}$ and $X$.

$$
D\left(x, X_{0}, X\right)=\left\{\begin{array}{cc}
\rho(x, X)-\rho\left(x, X_{0}\right) & x \notin X_{0} \\
-1 & x \in X_{0}
\end{array}\right.
$$

The value of the position $\mathrm{D}\left(\langle x, y\rangle, X_{0}, X\right)$ is the relation between the region $\langle x, y\rangle$ and regions $X_{0}$ and $X$.

$$
\left.D(<x, y\rangle, X_{0}, X\right)=\left\{\begin{array}{cc}
\rho(\langle x, y\rangle, X)-\rho\left(<x, y>, X_{0}\right) & x \notin X_{0} \\
-1 & x \in X_{0}
\end{array}\right.
$$

The template is used to format your paper and style the text. All margins, column widths, line spaces, and text fonts are prescribed; please do not alter them. You may note peculiarities. For example, the head margin in this template measures proportionately more than is customary. This measurement and others are deliberate, using specifications that anticipate your paper as one part of the entire proceedings, and not as an independent document. Please do not revise any of the current designations.

\section{Basic associative function}

Based on distance and value of the position, basic associative function of point $x$ in regard to classical domain region $X_{0}$ and festival field region $X$.

$$
k(x)=\frac{\rho\left(x, X_{0}\right)}{D\left(x, X_{0}, X\right)}
$$

And the basic associative function of region $\langle x, y\rangle$ in regard to classical domain region $X_{0}$ and festival field region $X$.

$$
k(<x, y\rangle)=\frac{\rho\left(\langle x, y\rangle, X_{0}\right)}{D\left(\langle x, y\rangle, X_{0}, X\right)}
$$

The extenics associative function $k$ is used to calculate the degree of the associability of relationship between a point and a region, or between a region and a region. If $k<0$, the degree of the associability of $x$ is interpreted as not related to $X_{0}$. If $k \geqq 0$, the degree of the associability of $x$ is related to $X_{0}$. When $-1<k<0$, then the current situation of $x$ is not related to $X_{0}$, but it can be transferred to be related to $X_{0}$.

\section{RISK MULTIDIMENSIONAL MEASURE BASED ON IMPROVED EXTENICS METHOD}

Suppose a risk has $n$-dimensional measurement indicators, each indicator has $m$ risk levels, correlation function value of risk measurement indicator $i(1 \leqq i \leqq n)$ belong to risk level $j(1 \leqq j \leqq m)$ is $k_{i j}$, take the risk level of $\max \left\{k_{i j}\right\}$ as the calculation basis to calculate the final risk degree, which are the traditional extenics method, when the value $k_{i j}$ difference is small, the results credibility being questioned. In view of this, this article will try to improve the extenics methods.

Correlation function value of risk measurement indicator $i(1 \leqq i \leqq n)$ belong to risk level $j(1 \leqq j \leqq m)$ is $k_{i j}$, when $k_{i j}>0$, means that the risk indicators $i$ are in risk level $j$, so when calculating the single index correlation degree, taking $k_{i j}>0$ data to weighted summation, set up single indicator $i$ risk degree $R_{i}$ as

$$
R_{i}=\sum_{j=1}^{m} k_{i j} \times \frac{a_{i j}+b_{i j}}{2} \quad\left(k_{i j}>0\right)
$$

Where, $a_{i j}, b_{i j}$ are the $j(1 \leqq j \leqq m)$ risk level region ends elements, $\left(a_{i j}+b_{i j}\right) / 2$ representing the region intermediate value, $R_{i}$ is the risk degree of single indicator. When $k_{i j}<0$, $R_{i}$ is not participate into calculation. Formula (12) considering the extent of single risk indicator $i$ belong to different risk levels, rather than based on $\max \left\{k_{i j}\right\}$ to interpret risk level.

After getting the single degree $R_{i}(1 \leqq i \leqq n)$ of risk indicator $i$, risk degree of multi-dimensional risk indicators should be integrated. Based on geometric mean and the arithmetic mean consideration, the following formula can be used to calculate the synthesized risk degree:

$$
\begin{aligned}
& R=\frac{1}{2}\left[\left(\prod_{i=1}^{n} R_{i}\right)^{\frac{1}{n}}+\frac{1}{n} \sum_{i=1}^{n} R_{i}\right] \\
&=\frac{1}{2}\left\{\left[\prod_{i=1}^{n}\left(\sum_{j=1}^{m} k_{i j} \times \frac{a_{i j}+b_{i j}}{2}\right)\right]^{\frac{1}{n}}+\right. \\
&\left.\frac{1}{n} \sum_{i=1}^{n}\left(\sum_{j=1}^{m} k_{i j} \times \frac{a_{i j}+b_{i j}}{2}\right)\right\} \quad\left(k_{i j}>0\right)
\end{aligned}
$$

Formula (12) and (13) improved the extenics methods, the correlation function and affiliation between multidimensional risk indicators to classic matter-element region (risk level region) and festival field matter-element region (risk indicators value region) were considered. The geometric mean and arithmetic mean of single indicator risk degree was integrated to calculate the final comprehensive risk degree. Relative to consider only the multidimensional risk index value, the proposed method offered the risk level standard, and analyzed the correlation function between index value to risk level standard region, used the product of plus correlation function value and region middle value to calculate the single index risk degree, and finally used the geometric mean and arithmetic mean to calculate the comprehensive risk degree, which is the improved use of extenics method.

Defined risk emergence and risk entropy, where risk emergence is the process characterization of causation nodes risk state being springed in complex system, and 
risk entropy is the quantificational measurement of springed risk state. On the basis of complex dynamical networks nodes risk state synchronization angle of view, analyzed the dynamical mechanism of risk emergence. By ameliorated the Extenics method, offered the causation node compositive risk degree calculational model under multidimensional risk measure structure, then provided the accident causation node marginal risk entropy measure method, combined the causation node coupling risk entropy based on Copula function, constructed the topper causation node risk entropy calculate model, and offered the resolution formula based on Jaynes entropy. Finally, the Arena software was used to simulate the risk emergence in topper causation node, the result shows that the increase of risk emergence in topper causation node obeys to multidimensional non-linear characteristic.

\section{CONCLUSIONS}

This paper presented a multi-dimensional measurement method for same type of risk, from the perspective of improving extenics method to achieve the multidimensional measure of risk, but for different types of risk, such as in complex equipment development process, there are technical risk, cost risk, schedule risk and management risk, which can be carried out on the basis of the above measure method, further integration of different types of risks, entropy can be used to unify the measure, which is the future research direction.

Next, we will synthetically consider the static network structure, expert experience and dynamic simulation data, offer an accident causation nodes importance evaluation arithmetic based on entropy weight concentrates multi-dimensional data; based on spare risk treatment resource and treatment velocity, the Arena software will be used to compare the compositor of the four different nodes importance evaluation results, and the entropy weight concentrates multi-dimensional data method gained the best result. Based on such evaluation result, simulate the emergent constraint entropy deploy process, when the emergent constraint entropy was engrossed by nodes according to its important degree, the system gain the best risk treatment effect. And increase the amount of emergent constraint entropy, the risk treatment capability of system can be heightened observably.

\section{ACKNOWLEDGMENT}

This work was supported by national natural scientific fund of china (Grant No. 71171199), and some professors in Engineering University of Armed Police Force of China helped me a lot, I thank all of them here.

\section{REFERENCES}

[1] Ma Xin,Li Ke-Ping,Luo Zi-Yan,and Zhou Jin. Analyzing the causation of a railway accident based on a complex network[J]. Chin. Phys. B Vol. 23, No. 2 (2014) 028904-1-7.

[2] J.H. Saleh, K.B.Marais, E.Bakolas, R.V.Cowlagi. Highlights from the literature on accident causation and system safety: Review of major ideas, recent contributions, and challenges[J]. Reliability Engineering and System Safety 95 (2010) 1105-1116.

[3] Jinqiu $\mathrm{Hu}$, Laibin Zhang, Lin Ma et al . An integrated method for safety pre-warning of complex system[J] . Safety Science , 2010 , 48 ( 1 ) : 580-597

[4] Qureshi Zahid H . A review of accident modelling approaches for complex critical sociotechnical systems . http://www.dsto.defence.gov.au/corporate/reports/DSTO TR-2094.pdf , 2008-01-30 .

[5] Nancy G. Leveson. Applying systems thinking to analyze and learn from events[J]. Safety Science, 2011 (49):55-64

[6] Coleman, M. E., \& Marks, H. M. (1999). Qualitative and quantitative risk assessment. Food Control, 10:289-297.

[7] T.M. WILLIAMS.Two-Dimensionality of Project Risk. International Journal of Project Management, 1996, 14(3):185-186.

[8] Wei Liu. ANALYSIS OF RISK MEASURES AND MULTIDIMENSIONAL RISK DEPENDENCE[D]. UNIVERSITY OF TORONTO,2008

[9] Sharpe W F. Capital asset prices: a theory of market equilibrium under conditions of risk[J]. Journal of Finance, 1964,19:425-442.

[10] Rasmussen, J. \& Svedung. X.. Proactive Risk Management in a Dynamic Society. Karl, Sweden: Swedish Rescue Services Agency,2000.

[11] Cai Wen, Yang Chunyan, Wang Guanghua. A New Cross Discipline-Extenics. SCIENCE FOUNDATION IN CHINA, 2005 13(1) :55-61.

[12] Ying-Chen Lee, Nobuyoshi Terashima. An Extenics-based Intelligent Distance Learning System. International Journal of Computer Science and Network Security, 2011,11(5):57-63. 